\title{
CHEMICAL COMPOSITION OF THE ESSENTIAL OIL OF Hyssopus cuspidatus FROM XINJIANG, CHINA
}

\author{
Palida Ablizl*, Yuanyuan Cong, Mirigul Musa, \\ Yan Zhu, and Rena Kasimu
}

UDC 547.913

The genus Hyssopus (Labiatae), consisting of about 15 species, is distributed in Central Asia, South Europe, and North Africa. In China, there are only two species, Hyssopus cuspidatus Boiss and H. latilabiatus C. Y. Wu et H. w. Li, all growing in Xinjiang [1]. Both of them have been used as nectar source plants and medicinal plants. In Xinjiang, H. cuspidatus Boriss. has also been used in traditional Uygur medicine in the treatment of cough, asthma, bronchitis, trauma, and rheumatism $[2,3]$. As the major Hyssopus resource for medical use in China, there are few reports on the essential oil of Hyssopus cuspidatus previously.

We identified a total of 36 compounds in the hydrodistilled essential oil of Hyssopus cuspidatus from Xinjiang, China, with germacrene D (18.67\%), hexadecanoic acid (17.53\%), germacrene B (15.61\%), $(+)$ trans-caryophyllene $(8.04 \%)$, and $(+)$ spathulenol $(4.11 \%)$ as the main components (Table 1). Further investigation of Hyssopus cuspidatus essential oil is still needed.

TABLE 1. Composition of the Essential Oil of Hyssopus cuspidatus

\begin{tabular}{|c|c|c|c|c|c|}
\hline Compound & $\mathrm{Rt}, \min$ & Content, $\%$ & Compound & $\mathrm{Rt}, \min$ & Content, $\%$ \\
\hline 1,8-Cineole & 28.51 & 0.23 & Cadinene & 64.19 & 0.52 \\
\hline Linalool & 34.18 & 0.39 & Elemol & 66.19 & 0.37 \\
\hline 1-Methyl-5,6-diethenylcyclohexene & 37.58 & 0.34 & Isoxadinene & 67.79 & 0.81 \\
\hline Camphor & 38.15 & 0.62 & $(+)$-Spathulenol & 68.07 & 4.11 \\
\hline 4-Terpinene & 40.81 & 0.33 & Caryophyllene oxide & 68.37 & 3.05 \\
\hline Pulespenone & 45.23 & 0.33 & Cetane & 74.63 & 1.06 \\
\hline Cumaldehyde & 45.64 & 0.84 & 2-Lanolin alcohol-1 & 75.58 & 0.48 \\
\hline 4-Methy-3-isopropenyl-4-vinyl-1-cyclohexene & 49.40 & 0.68 & Tetradecanoic acid & 78.12 & 0.65 \\
\hline Copaene & 54.96 & 0.30 & Octadecane & 80.08 & 0.60 \\
\hline$\beta$-Bourbonene & 55.57 & 0.67 & 6,10,14-Trimethyl-2-pentadecanone & 82.29 & 6.08 \\
\hline$\beta$-Elemene & 55.81 & 0.60 & Nonadecane & 85.28 & 0.64 \\
\hline trans-Caryophyllene & 58.02 & 8.04 & Hexadecanoic acid & 88.72 & 17.53 \\
\hline Farnesene & 59.79 & 2.43 & Heliannuol & 90.56 & 1.19 \\
\hline$\alpha$-Caryophyllene & 60.36 & 1.14 & Heneicosane & 94.93 & 1.43 \\
\hline Germacrene D & 62.02 & 18.67 & 4,4-Dimethyl-1-heptene & 95.26 & 0.32 \\
\hline Ledene & 62.58 & 0.51 & Octadecatrienoic acid methyl ester & 96.93 & 2.00 \\
\hline Germacrene B & 62.92 & 15.61 & Tricosane & 104.12 & 1.12 \\
\hline$\beta$-Bisabolene & 63.44 & 1.14 & Ledol & 108.43 & 0.5 \\
\hline
\end{tabular}

\section{REFERENCES}

1. Z. Y. Wu and X. W. Li, Flora of China, M. 242, 66 (1977).

2. Y. M. Liu, Pharmacography of Uighur, M. 423 (1999).

3. J. B. Ding, X. H. Wu, and Y. N. Wang, Xinjiang J. Trad. Chin. Med. [J], 9, 3 (2002).

School of Pharmaceutical Sciences, Xinjiang Medical University, 830054, Urumqi, Xinjiang, P. R. China, fax: 0991 4362473, e-mail: palida3345@yahoo.com.cn. Published in Khimiya Prirodnykh Soedinenii, No. 3, p. 374, May-June, 2009. Original article submitted November 5, 2007. 